\title{
Re-Design of Transaction Rules with Socialist Thought as the Core
}

\section{Shichong Wei, Xiong Li}

School of Information Technology, Shanghai Jianqiao College, Shanghai, China

Email address:

Wsc96710@126.com (Shichong Wei),17032@gench.edu.cn (Xiong Li)

\section{To cite this article:}

Shi Chong Wei, Xiong Li. Re-Design of Transaction Rules with Socialist Thought as the Core. Science Innovation. Vol. 7, No. 2, 2019, pp. 76-80. doi: 10.11648/j.si.20190702.15

Received: April 13, 2019; Accepted: May 27, 2019; Published: June 15, 2019

\begin{abstract}
Reverse trading uses an incentive mechanism similar to the college entrance examination. Your contributions to society are converted into points according to time, just as students get scores through hard work, and the priority of products and services is judged by your total points, just like the university enrollment mechanism. You can't guarantee that others won't "work hard together and secretly" and you still need to make constant contributions to society to keep your priority in getting products and services.
\end{abstract}

Keywords: Transaction, Economic System, Socialism, Economic Distribution

\section{以社会主义思想为核心重新设计交易规则}

\section{韦诗肿, 李雄}

上海建桥学院信息技术学院, 上海, 中国

\section{邮箱}

wsc96710@126.com(韦诗羽)，17032@gench.edu.cn（李雄）

摘要: 逆向交易采用的是类似高考的激励机制, 你为社会做出的贡献按时间付出换算成积分, 就如学生通过努力获得 的分数, 获得产品和服务的优先程度按你的总积分来判断, 就如大学的招生机制那样。你无法保证别人不会“明上说一 起不学习, 背地偷偷刻苦勤奋”, 你依然需要不断为社会做出贡献, 来保持你在获得产品和服务的优先程度。

关键词: 交易, 经济制度, 社会主义, 经济分配

\section{1. 引言}

社会主义和市场经济相结合一直以来都是世界难 题，社会主义和资本主义是当今社会上两大主义，然而 社会主义却从来没有自己的一套反映自身中心思想的 经济制度。马克思和恩格斯对社会主义发展出了他们的 理论体系, 亦认为社会主义社会是资本主义社会向共产 主义社会过渡的社会形态, 那么社会主义相对资本主义 应该展现出明显的优越性。社会主义和资本主义最为本
质的区别是生产资料所有制的区别, 经济包括着人类的 生产、储蓄、交换、分配的各项活动; 生产是这一动态 的基础, 分配是这一动态的终点。如果社会主义没有一 套属于自己的经济制度来描述经济中社会主义式的分 配, 那么目前的社会主义也不会是完整的。公平与正义 是人类永恒追求的社会发展目标, 只有在最基本的经济 体制内改革才能还原社会主义公平与正义的最终目的 [1]。

这社会上有很多公益类项目, 有一些是并不盈利但 对社会有很大积极影响的, 而这却和目前的经济制度产 
生了矛盾, 目前的经济制度下任何项目一定需要有人出 钱才能运作起来, 要有盈利才能实现, 而公益项目只能 靠个人或组织以慈善的形式运作, 让大众广泛受益和没 有盈利产生了矛盾。需要资金到位才能达成交易这一门 槛也会限制其生产力, 当甲方有需求但资金不到位，乙 方也不能开始生产。社会企业在进行商业运作的同时还 担负着改善社会问题的使命，如何兼顾两者值得探讨。 在制度逻辑分析框架下, 以上海市 $\mathrm{L}$ 机构为案例, 分析 发现社会企业中存在两种并行的制度逻辑一一市场逻 辑和公益逻辑, 这两种制度逻辑的互动与冲突导致社会 企业目标难以兼顾[2]。

战争一直是人们难以解决的问题，问题的起因涉及 软性的宗教, 种族问题, 也涉及硬性的利益问题, 如果 贫富差距过于悬殊上升为社会主要矛盾, 同样有可能导 致社会分崩离析[3]。如果要熄灭利益和贫富差距造成 的硬性战争导火索, 就需要一套正和博弯为主的新经济 体制的建立。若能将整合博弯的经济体制实现，国际内 所有国家都会开始同步发展共同富裕。

人在生下来后, 就会开始适应这个世界的环境, 这 个世界上的秩序, 培养出适应制度的经验和处事, 间接 地影响了人的价值观道德观。很多人的价值观是在一个 大的环境背景下总结出的生存经验。所以一个能培养一 个人好的道德观与价值观的制度的建立尤为重要, 这能 从根本上杜绝不好的社会现象。资本主义下的经济制度 是一套暗示剥削才能生存的体系, 虽然本质是交换, 却 暗示着相互掠夺, 只有掠夺才能生存的生存道理, 让人 们的内心深处普遍扎根着不安全感, 而这样一种生存道 理会在特定情况下激发出人们损人利已的动机, 消极的 影响了人们对待人和事的态度。追根到底, 目前的经济 制度以零和博弯为主, 才引起了上述经济制度对世界的 影响, 所以接下来本文会探究一种以正和博弯为主的社 会主义式经济制度。

人与经济是一体的, 人离不开经济, 经济也离不开 人。人是通过其活动所获取的物质生活资料来确保自己 的生存的, 直接以经济作为自己的生存方式, 换句话说, 人不仅要依靠经济而生存, 而且就在经济中生存[4]。

\section{2. 方法}

\section{1. 价值判定}

对于产品价格的判定，就算不看实际投入的成本， 不同的人在其价格上也会由于禀赋效应[5]产生不一样 的看法, 不能让所有人都信服每一件产品的价格。所以 就需要一套新的价值判定方法判定出每样产品让人们 信服的价格。

社会是由人和自然组成的, 人们通过智慧和劳动, 将自然资源加工成消费资料，从这个角度来看的话，任 何一样消费资料, 都源于大自然, 归大自然所有, 而人 们将自然资源加工为消费资料提供给别人时, 付出的只 有时间。所以任何消费资料的价值可以用时间来衡量,
因为为了更容易统计价值, 故可将时间按比例转化为积 分的形式以统计。

由于产品和服务都按照时间做价值判定是一项复 杂而庞大的步骤, 而且重新按时间投入决定产品和服务 价值会重构社会收入分配，与利益受损一方建立敌对关 系, 在前期依然按照目前货币的市场定价来决定产品和 服务的价值, 这并不会影响体系最终目的。

相关规则:

1. 一个人将自然资源加工成产品, 投入了时间 $\mathrm{N}$, 那么 这件产品价值为 $\mathrm{N}$

2. 乙方给甲方提供服务后, 体系自动承认乙方的投入时 间

3. 一件产品从资源采集, 到资源加工, 到物流货运, 分 别花费了时间 $A, B, C$, 那么产品总价值 $\mathrm{N}=\mathrm{A}+\mathrm{B}+\mathrm{C}$ 4. 一件产品从资源采集, 到资源加工, 到物流货运, 分 别花费了时间 A, B， C, 那么产品提供出去后, 体系 分别承认资源采集, 资源加工, 物流货运贡献了价值

$\mathrm{A}, \mathrm{B}, \mathrm{C}$

\section{2. 价值承认}

逆向交易与交易之间的区别在于逆向交易遵从社 会主义思想, 主张整个社会应作为整体, 由社会拥有和 控制产品、土地、资产等, 其管理和分配基于公众利益, 逆向交易中每个人都汇聚作社会的一部分而不是单独 个人, 每个人的甲方都代表社会, 当满足了他人的需求 后就是为社会产生了有效价值, 系统就会给予相应积 分, 承认其贡献。

逆向交易秉承着社会主义的思想,将社会的生产资 料公有化, 社会生产的产品和服务最终目的是分配给每 一个需要的人而不是为了卖出更高价钱获得更高利润, 人们在获取产品和服务时, 所持有积分和货币有所不 同，积分并不是用来交换和消耗的存在，而是用来做获 取产品和服务时用户优先度权重的排名砝码, 所持有的 积分越高优先权越大。

相关规则:

1. 乙方的限量服务和产品按甲方富有程度从高到低按 顺序提供

2. 甲方获得服务与产品后，总的时间贡献值不会像钱一 样花出去, 甲方总的时间贡献值只是个参与排名的砝 码

3. 可持续生产的产品也按客户的富有程度按顺序和叠 加的方式提供

4. 排名靠前的用户可以

\section{3. 乙方规则}

\subsection{1. 企业}

创造的价值=交易总数* (生产时间转化的积分数+ 交易额外积分）

交易上限 $=$ 用户平均交易次数 $*$ (用户总数 + 最近特 定周期新增用户数) 


\subsection{2. 职员}

回报 $=$ 基础劳动时间*职位学历要求对应回报加成 +所在企业的受益客户和总价值承认的分配。

\section{4. 甲方规则}

\subsection{1. 供小于求情况下}

获得顺序按个体总财富大小顺序排序，总财富 $=$ 总 积分*用户总数。

\subsection{2. 供大于求情况下}

假设公司A有1000名客户，公司持续生产产品，此 刻能提供富有度第 100 名客户 2 个产品 $\mathrm{A}$, 而这名客户却 要了 5 个产品, 那么多出来的 3 个产品, 从倒数前 3 富有 度的用户中每人减去 1 个此刻可以得到的产品 $\mathrm{A}$ 数量。

假设公司 $\mathrm{A}$ 有 1000 名客户, 公司持续生产产品, 此 刻能提供富有度第 100 名客户 2 个产品B, 而这名客户目 前表示不需要, 这名客户富有度往后 2 名客户每人此刻 可以获得的产品 $\mathrm{B}$ 增加 1 个数量。

\section{5. 实现方法}

该制度比较依赖于线上的系统平台, 可以出品一款 APP, 以商业模式运作, 以时间衡量价值, 转化为积分 存在。扩大用户基数和行业覆盖面, 随着用户基数的增 长和用户的黏性提升, 完善管理体系, 使得体系里的虚 拟积分权威性尽可能接近货币。

前期涌入的客户推测出来大部分会是有时间有好 奇心的人, 这一类人群通常会是学生或者员工一类的人 群, 生产力比较低, 用户在体系中的定位相当于各种个 体户。产品在前期应通过包装而成为一款特色社交 APP, 通过互相实现愿望获得时间的贡献积分, 这相当 于还原社会交易初期各种个体户之间的小本交易。

中期, 客户数量达到了客观的数字, 产品可以开通 wifi号, 公众号等功能, 推测最先能吸引到一些成长型 小型企业, 工作室和本地店铺的加入, 在产品里提供活 动和福利, 赚取流量和用户。考虑到这阶段很多产业的 供应链还不完整, 很多需求链也没闭合, 产业就算为用 户提供了服务和产品, 现实中的成本在软件用户中也找 不到弥补, 所以这阶段用户可以混合一般的资金交易在 其中。

产业的供应链难完整, 需求链难闭合, 是产品在走 向后期最大的挑战之一。要解决这个问题, 除了公司主 动拉拢一些成长型企业进来以外, 产品还要从功能上还 原一个好的合作体系, 让产品有能自己“创造企业”的能 力, 通过活动激发用户的创造力和想象力, 让产品内部 拥有有别于现实社会的特有交易项目, 增加产品里积分 的不可代替性。

公司应寻找一种和电商平台互利共赢的方式, 获取 商家资源, 前期可以作为一款让商家处理滞销商品的平 台, 让用户按积分竞获产品, 用实在的物质收获吸引更 多用户，也为以后产品企业入驻提供了渠道。
公司主动拉拢一些大型企业和名人加入, 企业和企 业之间也可以在产品里通过产品的规则不需要钱也能 完成一些合作, 争取通过用户面的扩大完善好每一个产 品供应链, 闭合尽可能多的需求链, 使得用户慢慢的可 以在产品里获得一些生活中花钱才能获得的服务和产 品。

企业中包括了提供服务的企业和生产产品的企业, 其中生产产品的企业由于实物成本较提供服务的企业 更高而较难引入这样一套体系。运营方可以把广告的模 式进行改变, 如果有需要推广自己产品的企业打算在产 品内做广告, 可以就按照体系的规则来, 在产品的广告 获动里发布自己自设数量的产品, 每个月在这个月贡献 的价值最多的前面对应用户可以免费领取这些产品, 让 用户通过竞争才能得到这样的产品, 广告的效果也较 好。需要推广的公司需要在产品注册用户, 当参与广告 发布的企业越来越多, 需求链环节越来越多, 体系对实 体企业的吸引力就会慢慢提升, 也会对一般用户的吸引 力有了提升, 就可以对实体企业做了用户的留存。

公司应集思广益, 寻找更多聪明和有知识文化的人 一起探讨完善体户群和行业范围的方案。

为了避免后面加入的用户积分从零开始追不上先 加入的用户, 产品应具备一套新用户社会贡献积分转化 算法, 这套算法里着重提升学历学位的转换加成, 激励 一些高学历技术型人才的加入。

后期, 经过实践证明了体系的可行性, 摸索和完善 了体系之后, 开始向外国发展, 开始发售磁卡。规模越 来越大, 产品里虚拟的时间积分就越来越有权威性, 社 会的讨论越来越多, 政府就会开始重视。

\section{3. 结果}

这套制度是一套包含正和博弯的交易，应用于世 界, 世界的主题从掠夺的竞争变成贡献的竞争, 可以从 根本上积极影响了人们今后的处事动机。

当今交易制度以利益驱动生产力, 全凭一个人的资 金拥有量来判断一个人的社会贡献价值, 不能通过科学 的方法判断一个人的各方面素质和社会贡献潜力, 最后 衍生出了违反人道主义的剥削阶级, 过度在乎利益驱动 而与社会最根本的人性背道而驰, 忽视了社会最基本的 人道主义,收入分配不公是引发中国人经济增长与幸福 感背离的主要原因[6]。逆向交易化繁为简, 从卖方出 发, 首先承认卖方的贡献, 相反的对人们的需求追求降 低了门槛, 也为人们做出贡献的想法降低了实现的门槛, 让人们公平的竞争。

首先承认贡献者的贡献，取消需要的人资金的门 槛, 贡献者不再需要思考如何将自己产品卖出高价, 只 需要考虑自己想贡献的东西有没有人需要, 这样可以大 大提高资源的利用率，降低资源的浪费。

逆向交易从交易对象这方面将交易对象一律宏观 看待为社会的一部分, 却细化了付出回报的路径。交易 
体系里一个人想要做一家手机公司, 首先需要讲通投资 人投资, 投资人投资后两个人最后是否能得到回报要看 用户是否买单，就像是一场高难度奢博游戏; 逆向交易 里你想要做一家手机公司, 只要通过联系获得手机制造 厂家的服务, 获得运营公司的服务, 获得货运公司的服 务, 至于最后你的手机受不受大众青睐, 服务于你的公 司都不用担心, 因为它们接受帮助你的时候已经得到了 基本的贡献承认。这套体制在生产资料私有制的情况下 让人们获得生产资料的门槛大大降低, 避免了生产资料 私有制下, 资产阶级垄断经济权力的根本目的 [7]。

\section{4. 讨论}

逆向交易采用的是类似高考的激励机制, 你为社会 做出的贡献按时间付出换算成积分, 就如学生通过努力 获得的分数, 获得产品和服务的优先程度按你的总积分 来判断, 就如大学的招生机制那样。你无法保证别人不 会“明上说一起不学习, 背地偷偷刻苦勤奋”, 你依然需 要不断为社会做出贡献, 来保持你在获得产品和服务的 优先程度。

体系里每个人的总财富 $=$ 总积分 $*$ 用户总数, 总积 分相当于一个人目前为止赚到的所有钱, 用户总数相当 于目前为止交易过的所有客户，这样的财富计算方式会 让两个规模有一定差距的同行企业产生更大的财富差 距, 反而会增加贫富差距, 但体系按富有度排名分配劳 动资源, 财富的差距不会拉大其购买力差距, 就像考试 获得的成绩再高, 也只是第一名。当一个人在某个行业 取得很巨大的收获后, 再高的收获也不会再提升自己的 购买力, 但能保持就算不工作后长时间内购买力不会下 降。这一点就利用了人性, 这一设定是希望削弱财富顶 端的人生产欲望, 去除垄断现象, 加快每个领域产品的 更新换代，留给人们更多阶级提升机会。

这套经济制度里人们获得产品和服务不再有资金 限制, 但假若这样一套体系内不再参与工作的人数开始 有大幅度提升, 导致社会生产力下降的话, 每个人获得 产品和服务也会开始逐渐困难, 进而促使人们从新工 作, 最后社会的总生产力和总需求满足动态平衡。

这套经济制度里价值的判定纯粹按付出得时间来 判定的话, 会出现虚报时间成本的现象, 原本五分钟流 水线生产的产品或许会有人虚报时间成本为三十分钟, 如果没法解决信誉问题, 就会有套利, 就会有人凭空获 得高奉献值, 整个体系就会崩盘。

首先，逆向交易这套制度需要依附于一个互联平 台, 企业在平台上为产品的定价决定公开的产品上架频 率, 如果假定某企业只有一条生产线, 一条生产线只能 一次生产一样某产品, 时间成本五分钟的产品在平台上 一小时最多可交易十二次, 而时间成本三十分钟的产品 一小时最多交易两次, 无关公司实际的生产速度, 这样 保证了定价的高低不能凭空套取高利润, 再在这基础之 上，系统定义里加上“交易达成得到的回报加上一定的
交易提成”，一定时间内交易达成越多回报就会越高， 如果虚报了过低的时间成本, 交易量也受限于实际的生 产能力。

按时间计算成本做工资的话, 每个工作职位的时间 回报也不一样, 因为学历越高的人做的工作普遍也要比 学历低的人做的工作对社会价值更大。用时间计算工资 并不与这一事实存在矛盾, 假设在系统内某个人应聘进 入了某企业的一个职位, 系统会根据这个职位的学历要 求给予提成, 承认每个人工作之前的学习和自我提升的 时间成本。

相对于现在“你得到我就会失去”的交易制度，“你 得到我也没有损失”的逆向交易或许容易出现互相之间 进行没有实际交易内容的刷交易记录行为, 互相之间凭 空刷取价值积分, 这样的话就出现了不公平的财富增长 现象, 体系就会崩溃。结合了实际情况后, 社会上其实 很少会出现两个生产单位之间就能形成闭合需求链的 现象, 体系里可以设定成一个单位与另一个单位出现了 交易后这样一个供需关系就固定住, 被需求方不再对原 本的需求方有需求的交易。如果是实际的需求链闭合关 系, 就要提供反向需求关系的申请。当一个生产单位的 受众多到一定程度, 多少就会有人是生产单位也需求的 生产单位, 体系可以定义生产单位的需求用户中每一百 位用户里可以无条件建立一位反向需求关系, 之后还是 要根据现实的数据确定特定的行业多少位需求用户里 可以无条件建立反向需求关系。

公有制和私有制长期以来是并存的, 历史上是这 样, 在社会主义社会中也是这样, 自然在不同的社会形 态中公有制和所有制都有不同[8]。逆向交易制度中， 企业生产的产品跳过了价格限制按人们的资产总额按 顺序全部提供给需要的人，这是市场上产品的公有制; 人们获得的产品和企业获得的收入是自己的, 这是个人 财产的私有制。

信贷是当今经济制度中一个重要组成部分, 但信用 制度加大了资本主义危机的可能性, 信用在加快资本主 义经济发展的同时, 也似乎与资本主义危机根源融为一 体，投资扩张导致生产无限扩大[9]。逆向交易制度重 新调整了信用结构, 将每个人的信用建立在整个社会的 基础上, 最大化趋近实现人们无条件获取产品和服务, 从而可以舍弃货币的根本属性, 也取消了货币限制的生 产力, 让有需求的交易都接近无阻力的达成, 让社会总 生产力无限趋近了社会总需求, 大大避免了资本主义危 机的可能性。

在国际发展援助方面,资金问题是影响国际发展的 关键 [10]。若是实行逆向交易制度, 发起援助的国家会 当即获得价值的承认, 财富得到增加, 得到援助的国家 生产力上升, 人们生活水平也得到提升, 而发起援助的 国家财富大于得到援助的国家, 在很长一段时间内也能 优先获得得到援助的国家产品和服务, 实现双赢, 最后 世界各国都能同步应用最先进科技。 


\section{5. 结论}

社会主义不仅能拥有一套属于自己的经济制度,而 且这套经济制度相比资本主义经济制度更具优越性。目 前的逆向交易制度还停留在纯粹理论阶段, 到完美应用 于实际社会或许还需要很多改进, 但改进的步伐要始终 向着最终目的——实现正和博弯为主的社会; 实现社会 在不过度损耗环境下最大化生产和最充分化对总需求 的分配; 让人与人之间真正公平地追求财富。

\section{参考文献}

[1] 王亚妮,杨宏伟.共享发展是中国特色社会主义的本质要求 [J].思想政治教育研究, 2019, (1):18-22。

[2] 武静.社会企业如何兼顾公益与商业一一基于制度逻辑的 分析[J].北京社会科学, 2018,(10):119-128。
[3] 张继肖.人类命运共同体——破解零和博弯的新思路 [J].产 权导刊,2019, (1):48-51。

[4] 彭定光,陈新,钟立华. 论经济的为人性 [J].湖南师范大学社会 科学学报, 2019, (1):64-68。

[5] 薛求知. 将心理分析引入经济学—2017年诺贝尔经济学 奖获奖成果解读 $[\mathrm{J}]$. 企业管理, 2017, (12):6-10。

[6] 李铭,左官春. 经济增长与幸福感背离的制度经济学阐释 [J]. 华东经济管理, 2019, 第33卷(3):164-171。

[7] 虞崇胜,何路社.论生产资料所有制的效率与公平问题兼与吴敬琏、韩东屏商榷 $[\mathrm{J}]$. 宁夏党校学报, 2018, 第20卷 (1):11-19.

[8] 董辅衸. 消灭私有制还是扬弃私有制?[J]. 中国民 商,2019,(1)。

[9] 黄慧微,沈涛.马克思信用理论及当代价值探析[J].河北经贸 大学学报, 2019, (2):7-12, 72。

[10] 刘宁.国际发展援助的转变一目标、资源与机制 [J].国际展 望, 2019, (2):106-128，153。 